\title{
Concerning the generation of geomagnetic giant pulsations by drift-bounce resonance ring current instabilities
}

\author{
K.-H. Glassmeier ${ }^{1}$, S. Buchert ${ }^{1 *}$, U. Motschmann ${ }^{1}$, A. Korth ${ }^{2}$, A. Pedersen ${ }^{3}$ \\ ${ }^{1}$ Institute for Geophysics and Meteorology, Technical University of Braunschweig, Germany \\ ${ }^{2}$ Max-Planck-Institute for Aeronomy, Katlenburg-Lindau, Germany \\ ${ }^{3}$ European Space Research and Technology Centre, Noordwijk, The Netherlands
}

Received: 7 July 1997 / Revised: 19 August 1998 / Accepted: 21 August 1998

\begin{abstract}
Giant pulsations are nearly monochromatic ULF-pulsations of the Earth's magnetic field with periods of about $100 \mathrm{~s}$ and amplitudes of up to $40 \mathrm{nT}$. For one such event ground-magnetic observations as well as simultaneous GEOS-2 magnetic and electric field data and proton flux measurements made in the geostationary orbit have been analysed. The observations of the electromagnetic field indicate the excitation of an odd-mode type fundamental field line oscillation. A clear correlation between variations of the proton flux in the energy range $30-90 \mathrm{keV}$ with the giant pulsation event observed at the ground is found. Furthermore, the proton phase space density exhibits a bump-on-the-tail signature at about $60 \mathrm{keV}$. Assuming a drift-bounce resonance instability as a possible generation mechanism, the azimuthal wave number of the pulsation wave field may be determined using a generalized resonance condition. The value determined in this way, $m=-21 \pm 4$, is in accord with the value $m=-27 \pm 6$ determined from ground-magnetic measurements. A more detailed examination of the observed ring current plasma distribution function $f$ shows that odd-mode type eigenoscillations are expected for the case $\partial f / \partial W>0$, much as observed. This result is different from previous theoretical studies as we not only consider local gradients of the distribution function in real space, but also in velocity space. It is therefore concluded that the observed giant pulsation is the result of a driftbounce resonance instability of the ring current plasma coupling to an odd-mode fundamental standing wave. The generation of the bump-on-the-tail distribution causing $\partial f / \partial W>0$ can be explained due to velocity dispersion of protons injected into the ring current. Both this velocity dispersion and the necessary substorm activity causing the injection of protons into the nightside magnetosphere are observed.
\end{abstract}

Correspondence to: K.-H. Glassmeier

* Present address: Solar-Terrestrial Environment Laboratory,

Nagoya University, Japan
Key words. Magnetospheric physics (energetic particles, trapped; MHD waves and instabilities) - Space plasma physics (wave-particle interactions).

\section{Introduction}

Giant pulsations (Pg) are ULF-pulsations predominantly occurring in the morningside magnetosphere. They are characterised by their large amplitudes, and their striking quasi-monochromatic wave form with periods of typically $100 \mathrm{~s}$. Giant pulsations have been studied intensively in the past by e.g. Annexstad and Wilson (1968), Green (1979), Rostoker et al. (1979), Glassmeier (1980), and more recently by Chisham and Orr (1991), Chisham et al. (1992), Takahashi et al. (1992), Chisham and Orr (1994), Chisham (1996), and Chisham et al. (1997).

These studies show that giant pulsations exhibit a strongly localised pulsation wave field extending only $200-300 \mathrm{~km}$ in the north-south direction, and 600-700 $\mathrm{km}$ in the east-west direction at the surface of the Earth. Furthermore, Pgs are found to be characterised by rapid east-west phase variations with the azimuthal wave number, $m$, reaching values up to $m=-40$, where the negative sign indicates westward phase propagation. These characteristics led several authors to conclude that giant pulsation wave fields are propagated in the guided poloidal mode, first described in Dungey's (1954) seminal work on ULF-pulsation theory. Although Pg ground amplitudes are often smaller than those of Pc4-5 pulsations the ionospheric magnetic field of Pgs is much larger due to the screening effect of the atmosphere which scales exponentially with the azimuthal wave number (e.g. Hughes and Southwood, 1976; Glassmeier, 1984). This justifies calling this type of pulsation giant pulsations.

However, there is still considerable debate about the possible generation mechanism of Pgs. The fast 
azimuthal phase variation and indications toward an odd-mode wave structure led Green (1979), Takahashi et al. (1992), and others to suggest that a drift-wave instability of the magnetospheric ring current (e.g. Hasegawa, 1971) is the dominant plasma instability generating giant pulsations. Other authors such as Glassmeier (1980), Poulter et al. (1983), or Chisham and Orr (1991) favour the proton drift-bounce resonance instability (e.g. Southwood et al., 1969; Southwood, 1976) as a generation mechanism, and give arguments for an even-mode wave structure along geomagnetic field lines. However, no firm conclusion on the generation mechanism has been reached as yet.

Any future theory on the generation mechanism of giant pulsations requires solution of the following three problems: first, identification of the instability mechanism; second, identification of the mechanism generating the unstable plasma situation, and providing the plasma free energy to feed the instability; third, identification of the wave mode the unstable plasma couples to, and by which the disturbance is propagated within the magnetosphere, especially to the ground. Furthermore, any theory should be able to predict and explain the striking quasi-monochromatic wave form, the fast azimuthal phase variation, the strong spatial localisation, and the predominantly morningside occurrence.

Using correlated ground-magnetic and geostationary satellite observations of a giant pulsation event which occurred on October 4, 1978, 0645-0830 UT (0915-1100
MLT), these mentioned problems will be tackled and a conjecture is formulated which explains many typical characteristics of giant pulsations.

\section{Ground-magnetic observations}

During the period of the International Magnetospheric Study, 1976-1979, the University of Münster operated the Scandinavian Magnetometer Array (Fig. 1; for further details see Küppers et al., 1979), whose Earth magnetic field observations will be used in the present study. Figure 2 displays bandpass filtered $(60 \mathrm{~s}<T<300 \mathrm{~s})$ records of the $\mathrm{D}$-component of the Earth magnetic field variations in the time interval October 4, 1978, 0645-0830 UT along a north-south profile, profile 4 (see Fig. 1) of the Scandinavian magnetometer array (SMA). The station SOY at about $71^{\circ}$ geomagnetic latitude is the northernmost, and OUL at about $63^{\circ}$ latitude the southernmost station along the profile; station separation is about $120 \mathrm{~km}$.

From the data in Fig. 2 it can be seen that clear Pgactivity with a period of about $100 \mathrm{~s}$ was recorded in Northern Scandinavia in the time intervals 0650-0718 UT $($ MLT $=$ UT $+2.5 \mathrm{~h}$ ), and 0735-0818 UT. The $\mathrm{Pg}$ is most prominent at the stations MUO and PEL, where peak-to-peak amplitudes reach values of up to 20 $\mathrm{nT}$. To the north and south of these two stations the $\mathrm{Pg}$ becomes less visible and more irregular, especially at the

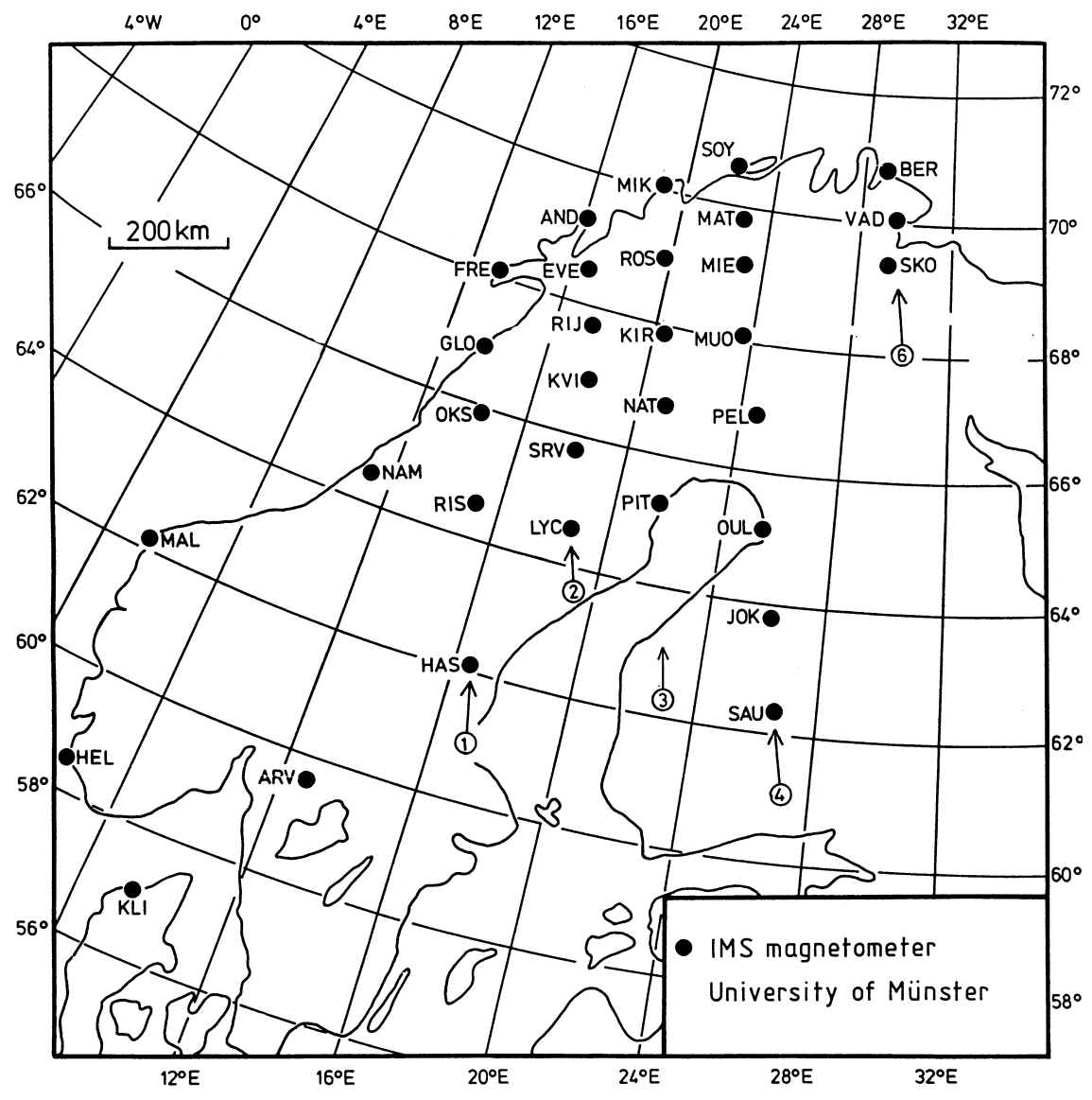

Fig. 1. Locations of the magnetometer stations of the Scandinavian magnetometer array in geographic coordinates. Encircled numbers with arrows denote different N-S profiles (after Küppers et al., 1979) 
$78-10-04$

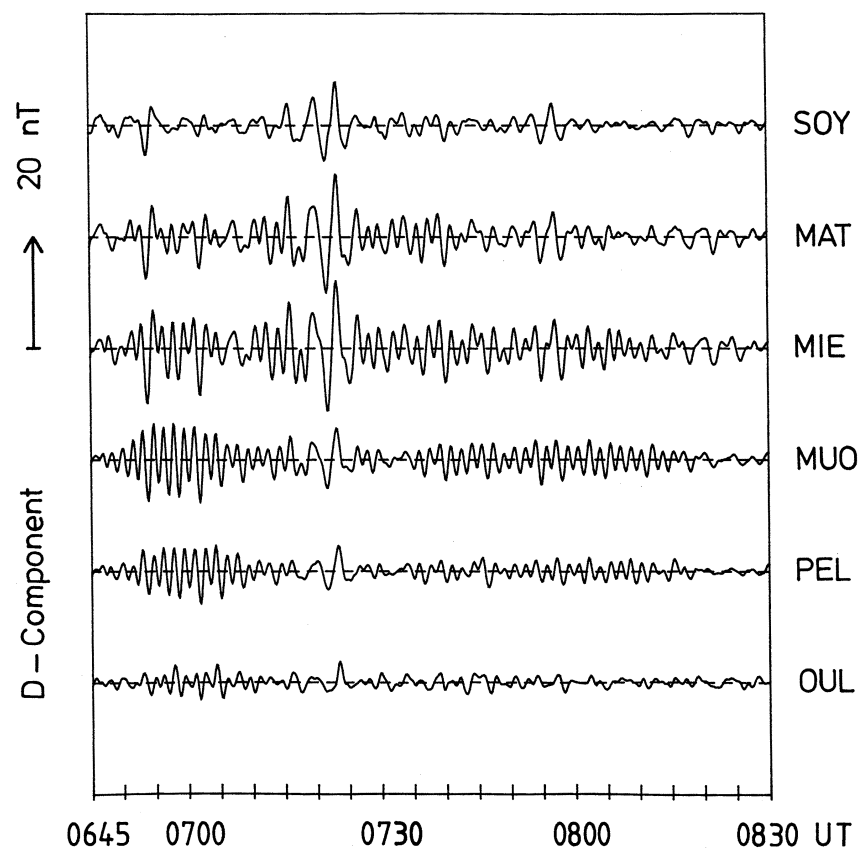

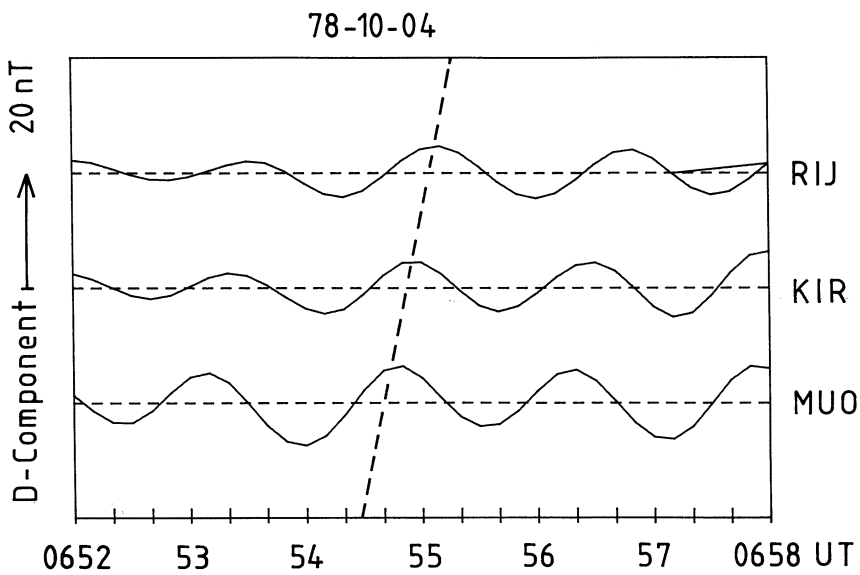

Fig. 3. Magnetic field variations recorded along the three east-west aligned stations RIJ, KIR, and MUO. Geomagnetic latitude of the three stations is $64.8^{\circ} \pm 1^{\circ}$. The dashed line connects corresponding maxima at the different stations

around the Earth of $\Omega_{A}=-2.4 \times 10^{-3} \mathrm{rad} / \mathrm{s}$. With the Pg-frequency $\omega=2 \pi / 100$ s this gives an azimuthal wave number $m=-26 \pm 7$, with the error estimate resulting from a sampling period of $10 \mathrm{~s}$ for the magnetic field observations used.

\section{Satellite observations}

For the giant pulsation event analysed satellite observations of the magnetospheric electromagnetic field as well as electron and ion flux measurements made onboard the European geostationary satellite GEOS-2 are available. GEOS-2 was launched into a geostationary orbit on July 14, 1978. During the time interval discussed the satellite was located at $37^{\circ}$ eastern geographic longitude; local time onboard GEOS-2 corresponds to UT $+150 \mathrm{~min}$. Field line tracing under moderate $\mathrm{Kp}$ conditions with a Tsyganenko-magnetospheric magnetic field model gives a nominal footpoint of the GEOS-2 field line close to the SMA station ROS (see Fig. 1). Thus the SMA and GEOS-2 are under nominal conjugate conditions. The geomagnetic longitude of GEOS- 2 in the equatorial plane is determined as $104^{\circ}$.

Having available satellite electric and magnetic observations is a significant achievement in trying to clarify the Pg generation mechanism. For example, inspection of the electromagnetic field observations allows us to determine the mode of oscillation of the observed event. Previous studies reached conflicting results with respect to this question. Green (1979), and more recently Takahashi et al. (1992) favour an oddmode, fundamental oscillation with anti-symmetric (symmetric) transverse magnetic field (electric field and field line displacement) variations. On the other hand, Glassmeier (1980), and Chisham and Orr (1991) give arguments for and strongly favour an even-mode, second harmonic standing field line oscillation with symmetric (anti-symmetric) transverse magnetic field (electric field and field line displacement) variations. An 
even mode (odd mode) can thus be identified in the magnetospheric equatorial plane as an oscillation exhibiting a node (an anti-node) of the electric field oscillation and an anti-node (a node) of the transverse magnetic field variation.

Figure 4 displays magnetic and electric field observations made onboard GEOS-2 in the time interval October 4, 1978, 0600-0800 UT. Magnetic field data are taken from the GEOS-2 fluxgate magnetometer (Candidi et al., 1974) and are represented in a dipole coordinate system. Thus, $\mathrm{B}_{r}$ denotes the radial component, counted positive outward, $\mathrm{B}_{\phi}$ the azimuthal component, counted positive to the east, and $\mathrm{B}_{\theta}$ completes the right-hand system. In all three magnetic field components no clear signal resembling, in any way, the characteristics of the giant pulsation event recorded at the ground can be identified. The regular, about $1 \mathrm{~min}$ oscillations in the $\mathrm{B}_{r}$ and $\mathrm{B}_{\phi}$ components are instrumental and due to a beat between the spin rate of $6 \mathrm{~s}$ and the data readout time of $5.5 \mathrm{~s}$. These oscillations do not indicate any natural variation. It is worthwhile noting that only the magnetic field magnitude increases (seen in the $\mathrm{B}_{\theta}$ component) at about 0615 and $0630 \mathrm{UT}$.

The electric field observations, however, display clearly the giant pulsation seen at the ground. The coordinate system used is again a dipole coordinate system with $\mathrm{E}_{\theta}$ approximating the magnetic field-aligned component. It does not completely vanish due to deviations from the $\theta$-direction from the actual magnetic field direction. Spiky perturbations at about $4 \mathrm{~min}$ past each hour are due to calibration sequences. The main $\mathrm{Pg}$ signal is recorded in the azimuthal component. At about 0630 UT a wave packet is seen lasting until about 0700 UT, when activity ceases for about $10 \mathrm{~min}$. From 0707 UT until 0737 UT the event is seen again. Some activity is also seen in the radial component. Spectral analysis reveals a pulsation period of $T=(110 \pm 5) \mathrm{s}$.

The correspondence between the period of the pulsation seen at the ground and in space as well as the clear packet structure of the event with almost the same packet length (see Figs. 2 and 4) strongly suggests that the same giant pulsation event has been observed. Moreover, it should be noted that the event at GEOS-2 already starts at $0630 \mathrm{UT}$, that is about $15 \mathrm{~min}$ earlier than at the ground. This is somewhat surprising as the nominal footpoint of the GEOS-2 field line is covered by the SMA viewing area. Thus, simultaneous onset of the perturbation was expected. We have, at present, no other explanation for this difference in onset time than questioning the validity of the field line tracing method used. As can be seen from Fig. 4 the magnetospheric magnetic field significantly changed at about 0630 UT,

October 4, 1978 DOY 277

GEOS 2

$5.5 \mathrm{~s}$ Averages

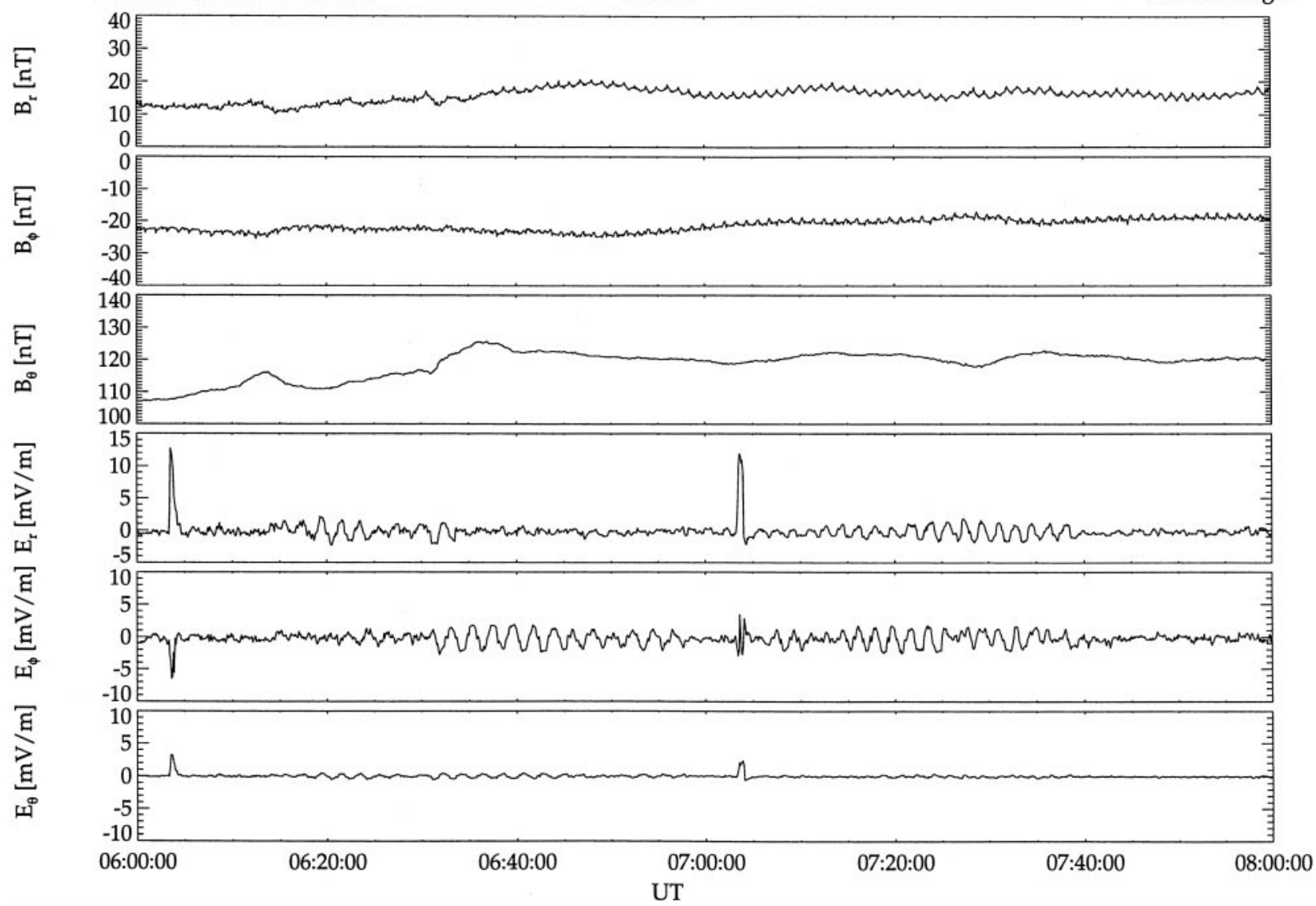

Fig. 4. Magnetic and electric field measurements made onboard the GEOS-2 satellite in the time interval October 4, 1978, 0600-0800 UT. The observations are represented in a dipole coordinate system 
a temporal variation which cannot be covered by any means with a Tsyganenko model. As the geographic position of GEOS-2 is about $20^{\circ}$ east of Scandinavia it is justified assuming that the true footpoint of the GEOS-2 field line is also located east of Scandinavia. Glassmeier (1980) and Poulter et al. (1983) reported westward group velocities of $200-300 \mathrm{~m} / \mathrm{s}$. Thus a delay time of $15 \mathrm{~min}$ between ground and satellite can easily be explained by an eastward shift of the footpoint of a few hundred kilometres. If the true footpoint is located east and if an azimuthal westward group velocity of a few hundred metres is assumed a delay of $15 \mathrm{~min}$ is easily explained.

The observation of transverse electric field variations in the magnetospheric equatorial plane and the absence of any correlated magnetic field variations indicates the presence of an odd mode standing field line oscillation. Whether the fundamental or any odd harmonic oscillation has been excited can be decided on grounds of the wave period. The electron density was measured at about $3 \mathrm{~cm}^{-3}$; assuming a plasma predominantly consisting of protons gives an equatorial mass density of $5 \times 10^{-21} \mathrm{~kg} / \mathrm{m}^{3}$. The observed large azimuthal wave number $\mathrm{m}$ suggests that a guided poloidal mode has been excited. For this type of mode Cummings et al. (1969) have calculated the eigenperiods for different harmonics and density indices $\mathrm{n}$ for density profiles $\rho(r)=\rho_{0}\left(r_{0} / r\right)^{n}$ along field lines passing the geostationary orbit, $\mathrm{r}_{0}=6.6 \mathrm{R}_{E}$. With this value for $\rho_{0}=5 \times 10^{-21}$ $\mathrm{kg} / \mathrm{m}^{3}$ and $\mathrm{n}=1$ eigenperiods of $\mathrm{T}=127 \mathrm{~s}$ and $\mathrm{T}=23$ $\mathrm{s}$ for the fundamental and the third harmonic guided poloidal mode, respectively, result. We may conclude that the observed electric field observations are due to a fundamental mode poloidal eigenoscillation. The observed predominantly azimuthal electric field variation corresponds to a predominantly radial magnetic field perturbation. Taking into account an ionospheric rotation by $90^{\circ}$ (e.g. Hughes, 1974; Glassmeier, 1984) a ground-magnetic perturbation dominating the D-variation is expected. This is indeed observed, which supports our conjecture of a guided poloidal mode having been excited.

However, the observed electron density may be too low because only part of the distribution function has been measured. Furthermore, the magnetospheric plasma is not a pure electron-proton plasma, but can also exhibit a significant oxygen component. Wedeken et al. (1984), for example, found a ratio of oxygen to proton ions of $1 / 15$ at the geostationary orbit. Singer et al. (1979) report even higher ratios. Using the Wedeken et al. (1984) value a mass density of $\rho_{0}=6.4 \times$ $10^{-21} \mathrm{~kg} / \mathrm{m}^{3}$ results. This corresponds to poloidal mode eigenperiods of $145 \mathrm{~s}, 41 \mathrm{~s}$, and $26 \mathrm{~s}$ for the fundamental, second harmonic, and third harmonic mode, respectively. A clear identification of the mode structure is thus not possible due to a lack of suitable mass density measurements.

Electron and proton observations made onboard the GEOS-2 satellite have been studied to find correlative signatures with the ground magnetic and the satellite electric field oscillations, identified as giant pulsations.
The particle measurements were performed with a magnetic spectrometer to separate electrons from ions (Korth and Wilken, 1978; Korth et al., 1978). Electron energy spectra are determined in 16 channels between 16 $\mathrm{keV}$ and $213 \mathrm{keV}$. Ions are analysed in 10 energy channels between 28 and $402 \mathrm{keV}$. Full energy spectra are obtained every $5.5 \mathrm{~s}$ in various pitch angle ranges. No identification of different ion species is possible. For the data interpretation we assume that most of the detected ions are protons.

From the data analysis of the particle instrument follows that only the ion observations significantly correlate with the giant pulsation event observed. Figure 5 displays proton flux measurements in six different energy channels from $28 \mathrm{keV}$ up to $114 \mathrm{keV}$ in the pitch angle range $25^{\circ}-35^{\circ}$. The flux shows appreciable variations in the different energy ranges. The most pronounced variations are increases of the flux at around 0700 UT and 0800 UT (see, for example, channel 5, 59-75 keV, in Fig. 5). These flux intensifications parallel the amplitude modulation of the $\mathrm{Pg}$ as observed in the ground-magnetic field and the GEOS-2 electric field observations. This indicates a possible relationship bewteen the modulation and flux increases. A more detailed interpretation, however, is not yet possible as the flux intensifications are local effects while the wave activity is caused by local plasma instabilities as well as waves which propagate in from other regions of the magnetosphere to the points of observations. Azimuthal Poynting flux observations are required to elucidate this point further. They are not available for the present study.

The proton fluxes also show velocity dispersion, that is the higher-energy protons arrive earlier than the lower-energy ones. This points toward the proton injection not occurring locally, but at some distance from the satellite. It should be noted that this modulation feature of the proton fluxes as well as the velocity dispersion can be observed for protons with pitch-angles up to $40^{\circ}$, but not at higher pitch-angles, for which no correlation between proton flux and ground-magnetic field variations has been found. Also, the proton flux observations do not indicate the presence of a significant proton temperature anisotropy which could give rise to a drift-mirror instability.

A possible injection mechanism for the observed proton clouds is substorm activity in the nightside magnetosphere. Figure 6 displays ground-magnetic field variations observed at the Canadian station Fort Providence (located at $292^{\circ}$ geomagnetic longitude) during the interval October 4, 1978, 0400-0900 UT (2100-0200 MLT), and indicates several substorms or substorm intensifications in the nightside magnetosphere at the same time the giant pulsation event is observed in the morningside (see Figs. 2 and 6). Substorm activity is therefore a candidate for the observed proton injections. Therefore, a relation between substorm activity and Pgs is suggestive, and such a connection has also been discussed by Glassmeier et al. (1992) and Chisham et al. (1992).

The observed velocity dispersion in the different energy channels can be used to determine the drift time 


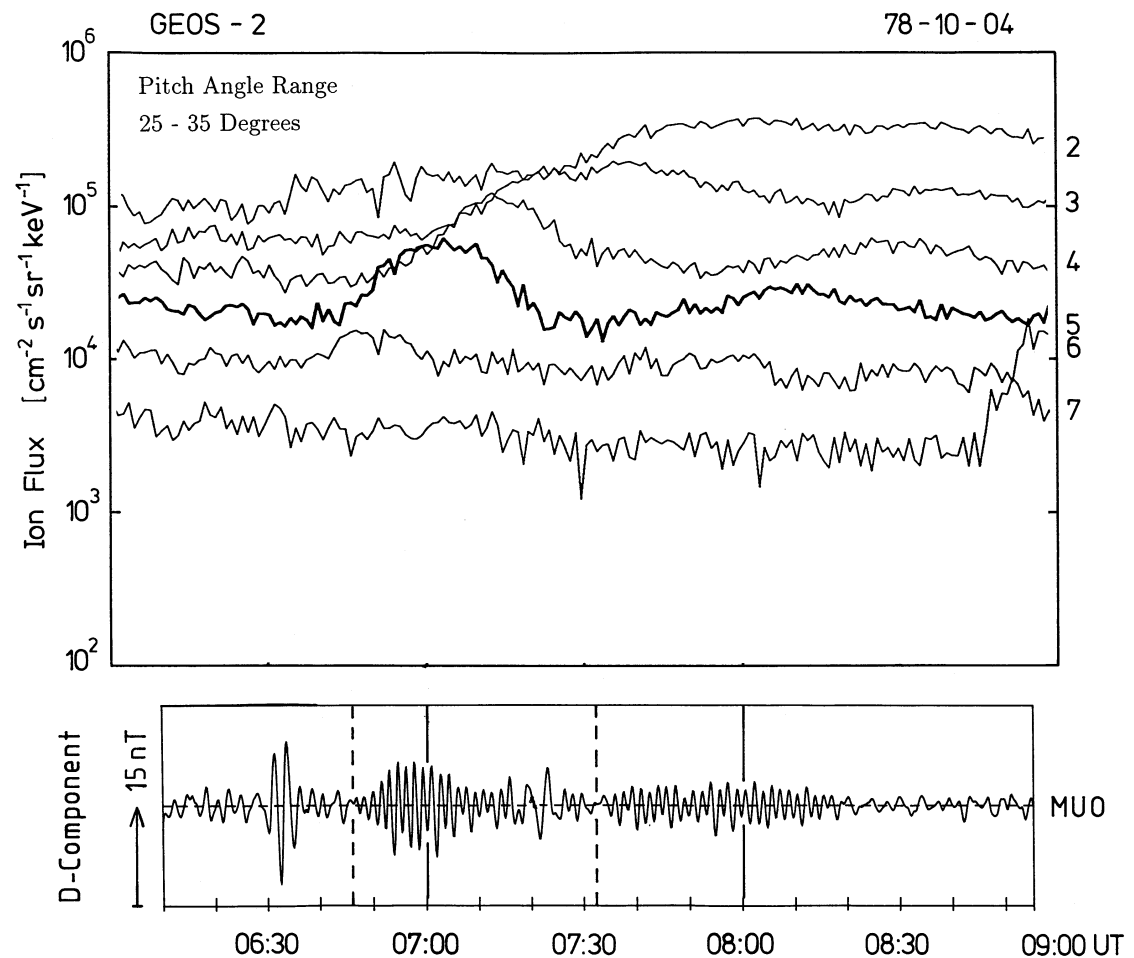

Fig. 5. Proton-Flux measurements made onboard the geostationary satellite GEOS-2 in the pitch-angle range $25^{\circ}-35^{\circ}$ on October 4, 1978, 06-09 UT (upper panel), and Earth magnetic field variations of the D-component at the station MUO (profile 4), close to the nominal footpoint of the GEOS-2 field line (bottom panel). The flux measurements are shown for six different energy channels; channel 2: 28-36 keV; 3: 36-49 keV; 4: 49-59 $\mathrm{keV}$; 5: 59-75 keV; 6: 75-98 keV; 7: 98-114 $\mathrm{keV}$. The magnetic field observations have been filtered in the range $60-300 \mathrm{~s}$ and also the drift periods of the protons. If $\phi$ is the actual longitude a proton has reached after the time $t$ at the injection point $\phi_{0}$, the relation

$\phi(t)=\phi_{0}+v_{\phi} \cdot t$

holds where $v_{\phi}$ is the azimuthal drift velocity. This velocity is negative for protons due to our definition of azimuthal variations counted positive eastward. According to Schulz and Lanzerotti (1974) $v_{\phi} \propto W$, the particle energy. With the difference in arrival time, $\delta t=t_{i}-t_{j}$, observed at the same place between the particles in energy channels $i$ and $j$, from the earlier equation

$t_{j}=\frac{W_{i} \cdot \delta t}{W_{j}-W_{i}}$

where for the particle energies $W_{j}>W_{i}$. The velocity dispersion as seen in Fig. 5 is emphasized most in the energy channels 3-6. With Eq. (2) a drift time of e.g. $t_{5}=2935 \mathrm{~s}$ for the $59-75 \mathrm{keV}$ protons results.

Assuming an injection point at around midnight (that is when Fort Providence exhibits substorm activity) the protons observed at GEOS-2 have thus drifted a distance of about $-188^{\circ}$ (the distance between Fort Providence and GEOS-2 in geomagnetic longitude) within this drift time. Accordingly a drift frequency $\Omega_{D, 5}=-(1.1 \pm 0.2) \times 10^{-3} \mathrm{rads}^{-1}$ may be determined from the particle observations. Here we allowed for an error of about $20 \%$ in the determination of $\Omega_{D}$ to account for the uncertainty of the determination of the injection point.

In a dipole field geometry gradient and curvature drift for $67 \mathrm{keV}$ protons would result in a drift frequency $\Omega_{D}=-0.8 \times 10^{-3} \mathrm{rads}^{-1}$. This value corresponds to the measured one. The proton flux measurements can be used to derive phase space densities as well. Figure 7 exhibits phase space densities at times 0655:18 and 0700:21 UT for the pitch angle range $5^{\circ}-15^{\circ}$. The most remarkable feature is a shoulder at an energy of about $60 \mathrm{keV}$, i.e. the energy range of channel 5. A similar shoulder is also seen at the same energy in other pitch angle ranges below $40^{\circ}$. We interpret this shoulder as indicating an unstable, bump-on-the-tail distribution. Thus, not only the correlation between Pg amplitude modulation and proton injection, but also the phase space densities point toward a causal relationship between the observed giant pulsations and proton injection.

\section{Drift-bounce resonance instability of the ring current plasma}

Hughes et al. (1978) reported a quasi-monochromatic wave event observed onboard the satellite ATS 6 in the afternoon sector of the magnetosphere and concurrent measurements of bump-on-the-tail protons phase space distributions. They convincingly argue that this unstable distribution via the bounce-resonance instability gives rise to the observed quasi-monochromatic wave. However, their bump-on-the-tail was observed at an energy of about $10 \mathrm{keV}$, and the wave was not identified as a giant pulsation.

Nevertheless, it is tempting to discuss the described GEOS-2 proton observations in terms of a drift-bounce resonance instability of the ring current plasma (Southwood et al., 1969). This instability might be discussed as an analogy of the cyclotron instability. In a mirror field 


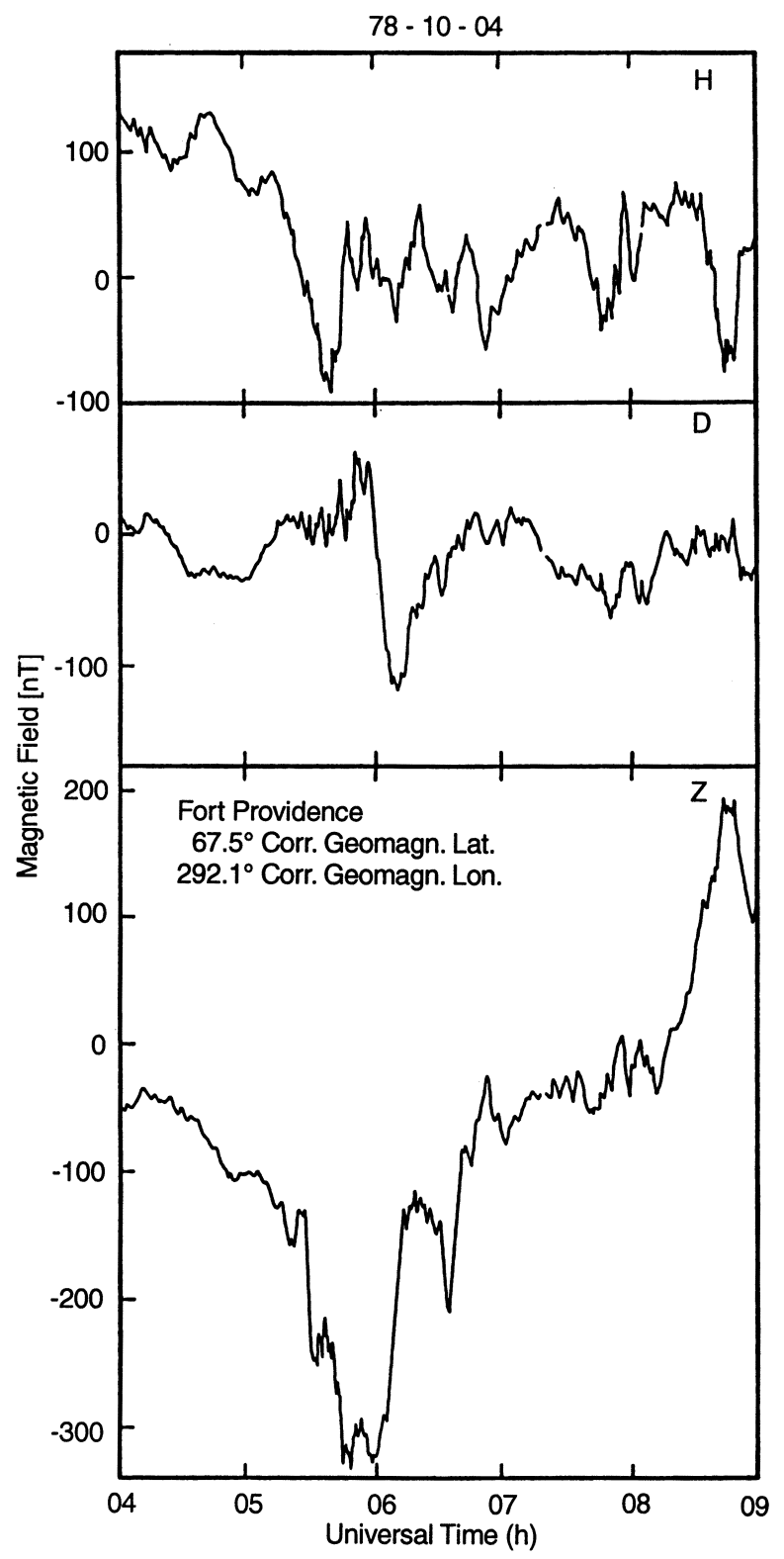

Fig. 6. Magnetic field observations, recorded on October 4, 1978, 04 09 UT, at Fort Providence, Canada (data courtesy G. Rostoker)

geometry a charged particle performs a bounce motion between the mirror points and an azimuthal drift motion in addition to the gyromotion around the local magnetic field direction. While the cyclotron instability is a resonant interaction between a plasma wave propagating along the ambient magnetic field and the gyrating particles, the drift-bounce resonance describes a resonant interaction between the drift-bounce motion of the particles and an azimuthally propagating wave. The change of the energy, $W$, of the particle with time is given by

$\frac{\mathrm{d} W}{\mathrm{~d} t}=q v_{\|} E_{\|}+\mu \frac{\partial b_{\|}}{\partial t}+q \vec{E} \cdot \vec{v}_{D}$

where $q$ and $\mu$ are the electric charge and the magnetic moment of the particles, respectively, and where $v_{\|}, b_{\|}$,

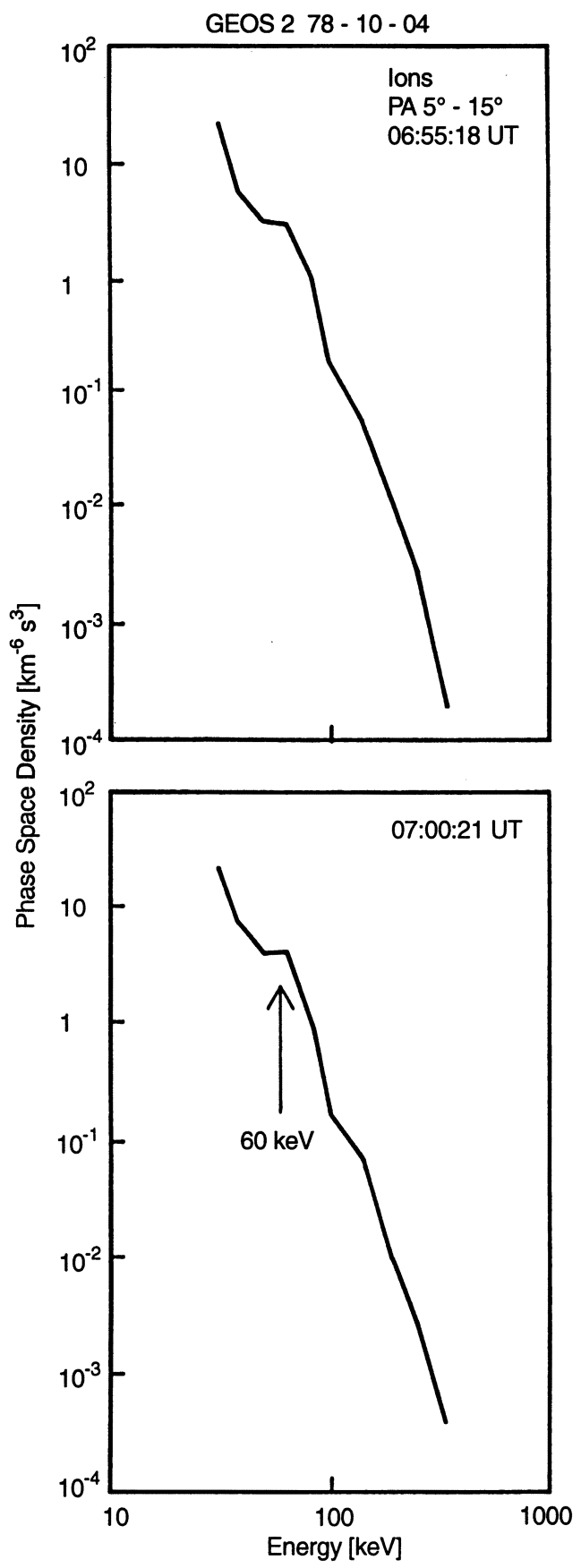

Fig. 7. Phase space density of energetic protons recorded onboard GEOS-2 in the pitch-angle range $5^{\circ}-15^{\circ}$ on October $4,1978,0655: 18$ and 0700:21 UT

and $E_{\|}$denote the particle velocity along the ambient magnetic field as well as the wave magnetic and electric field perturbations along the background field, and $\vec{v}_{D}$ is the unperturbed particle drift velocity. If the particle interacts with a purely transverse magnetohydrodynamic wave the first two terms in Eq. (3) vanish, and the interaction is via the third term only, i.e. in an inhomogeneous background field the azimuthal gradient and curvature drifts cause an interaction with the azimuthal wave electric field. 

1976)

The resonance condition is given by (e.g. Southwood,

$\omega-m \Omega_{D}-N \Omega_{B}=0$

where $\omega$ is the wave frequency, $m$ an azimuthal wave number, and $\Omega_{D}$ and $\Omega_{B}$ denote the particle drift and bounce frequencies, respectively. According to previous studies on the bounce resonance instability by e.g. Southwood et al. (1969), Southwood (1976), Glassmeier (1980), Southwood and Kivelson (1982), or Takahashi et al. (1992) $N$ is an integer. However, this is only true if the transverse component of the electric field perturbation is symmetric or antisymmetric about the magnetic equator. Any asymmetry allows $N$ to take any real value. Such an asymmetry can be due to asymmetry of the ionospheric Pedersen conductivity between the Northern and Southern Hemisphere. This occurs, for example, at the solstices, when one polar ionosphere is illuminated for a long time and the other is correspondingly in darkness. In this case the resulting standing wave has a quarter-wave form along the field line with clear asymmetry (Allan and Knox, 1978).

In more general terms the wave field along the ambient magnetic field must be regarded as a superposition of the fields of a standing wave and a travelling wave. Finite ionospheric conductivity causes a finite Joule heating in and thus a Poynting flux into the ionosphere (e.g. Allan and Knox, 1979; Glassmeier et al., 1984). If the Pedersen conductivities are equal in both hemispheres the Poynting flux into the ionospheres, integrated along the field line, must also be equal. This requires the existence of a null-point, where the local Poynting flux vanishes (Allan, 1982). For symmetric or antisymmetric electric and magnetic field perturbations this null-point is located right at the magnetic equator. However, if the ionospheric conductivities differ from each other this null-point is significantly shifted towards that ionosphere where the conductivity is larger. This implies a break-down of any symmetric or antisymmetric behaviour of the wave fields along the field line with respect to the magnetic equator. The null-point may be regarded as the new point of symmetry.

The effect of asymmetries on the resonance condition (4) can be demonstrated by the following simplified computation of the energy exchange per time between the wave field and a bouncing particle. The energy change of a particle due to its interaction with the electromagnetic field of the unstable wave is given by

$\delta \dot{W}_{B}(s)=q E_{\phi}(s) v_{D}(s) \exp (\mathrm{i}(m \phi-\omega t))$

where $q$ is the electric charge of the particle, $E_{\phi}(s)$ the arc length $s$ dependant azimuthal electric field component of the wave, $v_{D}(s)$ the arc length dependant azimuthal drift velocity, and $\phi$ the azimuth angle or azimuthal drift phase. The electric field may be written as

$E_{\phi}(s)=-\mathrm{i} E_{o} \exp \left(\mathrm{i} \frac{\alpha \pi}{L} s\right)$
This expression allows for asymmetric electric field variations with respect to the equatorial plane. Parameters $\alpha$ and $L$ describe this asymmetry and the arc length between the northern and southern footpoint of the oscillating field line, respectively. The arc length is counted positive from the northern mirror point. For $\alpha=1$ this functional dependance of $E_{\phi}$ allows to describe the odd-mode fundamental eigenoscillation. The quarter-wave is described by $\alpha=1 / 2$, while e.g. $\alpha=2 / 3$ describes an electric field variation which is asymmetric with respect to $s=L / 2$ and exhibits different values of the electric field at conjugate ionospheres. Such a mode would allow us to describe a situation with the null-point shifted towards one hemisphere.

For a particle bouncing in a dipole field one has

$s=l \cos \Omega_{B} t$

with $l$ the half-bounce path length, that is the rectified path distance between the northern and southern mirror points of the bouncing particle (e.g. Roederer, 1970, p.35); $s=0$ for the northern mirror point. This relation between arc length of the bouncing particle and time reflects the harmonic motion of the particle in a dipole field, provided $\cos ^{2} \theta \ll 1$, where the angle $\theta=\Omega_{B} t$ denotes the particle bounce phase (e.g. Schulz, 1991, p. 203). The general expression relating arc length and bounce phase is a more complicated nonlinear relation given by Eq. (150) of Schulz (1991). With this expression for the arc length $s$ the variation of the electric field as seen by a bouncing particle can be approximated by

$E_{\phi}(s) \approx-\mathrm{i} E_{o} \exp \left(\mathrm{i} \frac{\alpha \pi l}{L} \cos \Omega_{B} t\right)$

The arc length dependant drift velocity may be approximated by its bounce averaged value via

$v_{D}(s) \approx\left\langle v_{D}(s)\right\rangle=v_{D}$

and the drift phase is given by $\phi(t)=\Omega_{D} t$.

With these definitions one has

$\delta \dot{W}_{B} \approx-\mathrm{i} E_{o} v_{D} \exp \left(\mathrm{i}\left(m \Omega_{D}-\omega\right) t+\mathrm{i} \frac{\alpha \pi l}{L} \cos \Omega_{B} t\right)$

In this expression wave frequency $\omega$ and drift frequency $\Omega_{D}$ appear in a linear relationship with time $t$, while the bounce frequency $\Omega_{B}$ is related to time in a nonlinear cosine function term. To obtain a similar linear relation between bounce frequency and time as one has between drift frequency and time the expression $\exp \left(i \frac{\alpha \pi l}{L} \cos \Omega_{B} t\right)$ may be expanded into a Fourier series as done by e.g. Southwood (1976):

$\exp \left(\mathrm{i} \frac{\alpha \pi l}{L} \cos \Omega_{B} t\right)=\sum_{N=-\infty}^{N=+\infty} \gamma_{N} \exp \left(\mathrm{i} N \Omega_{B} t\right)$

It should be noted that Southwood (1976) actually expands the bounce phase $\theta$. The expansion coefficients $\gamma_{N}$ denote the spectral weight of each bounce phase harmonic function. 
Of particular interest is the bounce integrated energy transfer, that is;

$\delta W_{B}=\int_{0}^{T_{B}} \delta \dot{W}_{B} \mathrm{~d} t$

with the bounce period $T_{B}=2 \pi / \Omega_{B}$. Southwood's (1976) expansion results in:

$$
\begin{aligned}
\delta W_{B} \approx & q E_{o} v_{D} \\
& \left.\sum_{N=-\infty}^{N=+\infty} \gamma_{N} \frac{\exp \left(\mathrm{i}\left(m \Omega_{D}-\omega+N \Omega_{B}\right) \cdot t\right)}{\omega-m \Omega_{D}-N \Omega_{B}}\right]_{0}^{T_{B}},
\end{aligned}
$$

an expression approximated by Southwood [1976] as:

$$
\begin{aligned}
\delta W_{B} & \approx q E_{o} v_{D} \delta\left(\omega-m \Omega_{D}-N \Omega_{B}\right) \\
& \left.\times \exp \left(\mathrm{i}\left(m \Omega_{D}-\omega+N \Omega_{B}\right) t\right)\right]_{0}^{T_{B}} .
\end{aligned}
$$

It is at this point where the condition $N \in \mathscr{N}$ has been introduced by truncating the series over $N$. This truncation implies the following approximation:

$\exp \left(\mathrm{i} \frac{\alpha \pi l}{L} \cos \Omega_{B} t\right) \approx \exp \left(\mathrm{i} N \Omega_{B} t\right)$.

A different approximation allows relaxation of the condition $N \in \mathscr{N}$. The cosine dependence $\mathrm{s}(\mathrm{t})$ may be approximated by a triangle function via:

$s(t) \approx \begin{cases}\frac{l}{\pi} \Omega_{B} t & \text { if } 0 \leq t \leq T_{B} / 2 \\ \frac{l}{\pi}\left(1-\Omega_{B} t\right) & \text { if } T_{B} / 2 \leq t \leq T_{B} .\end{cases}$

For $t=T_{B} / 2$ this gives, for example, $s\left(t=T_{B} / 2\right)=l$. This triangular approximation provides one with a linear relationship between $s$ and $t$. It thus equals the already used relations between frequency and time as well as drift frequency and time. No expansion as used by Southwood (1976) is required. With this approximation the bounce integrated energy change reads:

$$
\begin{aligned}
\delta W_{B} & \approx-\mathrm{i} q E_{o} v_{D} \\
& \times \int_{0}^{T_{B} / 2} \exp \left(\mathrm{i}\left(m \Omega_{D}+n \Omega_{B}-\omega\right) \cdot t\right) \mathrm{d} t \\
& +\int_{T_{B} / 2}^{T_{B}} \exp \left(\mathrm{i}\left(m \Omega_{D}-n \Omega_{B}-\omega\right) \cdot t\right) \\
& \times \exp (i n) \mathrm{d} t
\end{aligned}
$$

where $n=\alpha l / L$ has been introduced. Integration of the first term on the right hand side of Eq. (17) gives

$$
\begin{aligned}
& \int_{0}^{T_{B} / 2} \exp \left(\mathrm{i}\left(m \Omega_{D}+n \Omega_{B}-\omega\right) \cdot t\right) \mathrm{d} t \\
& \left.=\frac{\mathrm{i}}{\omega-m \Omega_{D}-n \Omega_{B}} \cdot \exp \left(\mathrm{i}\left(m \Omega_{D}+n \Omega_{B}-\omega\right) \cdot t\right)\right]_{0}^{T_{B} / 2} .
\end{aligned}
$$

This expression maximizes, if $\omega-m \Omega_{D}-n \Omega_{B}=0$. Integration of the second term on the right hand side of Eq. (17) gives

$$
\begin{aligned}
& \int_{T_{B} / 2}^{T_{B}} \exp (\mathrm{in}) \exp \left(\mathrm{i}\left(m \Omega_{D}-n \Omega_{B}-\omega\right) \cdot t\right) \mathrm{d} t \\
& \left.\quad=\frac{\mathrm{i} \exp (\mathrm{in})}{\omega-m \Omega_{D}+n \Omega_{B}} \cdot \exp \left(\mathrm{i}\left(m \Omega_{D}-n \Omega_{B}-\omega\right) \cdot t\right)\right]_{T_{B} / 2}^{T_{B}} .
\end{aligned}
$$

This expression maximizes, if $\omega-m \Omega_{D}+n \Omega_{B}=0$. Thus, the resonance condition reads

$\omega-m \Omega_{D}-n \Omega_{B}=0$

with $n \in \mathscr{R}$. This is a proper generalization of the Southwood (1976) condition. Eqs. (4) and (20) agree, if $L=l$ and if $\alpha= \pm 1, \pm 2 \ldots$

This relation can be used to determine the azimuthal wave number

$m=\frac{\omega-n \Omega_{B}}{\Omega_{D}}$

where $m>(<) 0$ corresponds to an eastward (westward) propagating wave and a suitable value for $n$ has to be assumed. The protons under consideration have a pitch angle of about $30^{\circ}$, that is they mirror at an equatorial distance of about $35^{\circ}$ or $1 / 2=0.25 \mathrm{~L}$. Thus, we have $l / L \approx 0.5$.

To estimate the value $\alpha$ is more difficult. It requires knowledge of the ionospheric conductivity at the footpoints of those field lines perturbed by the Pg pulsation considered. Measurements of the conductivities are not available. However, using a Tsyganenko 89 magnetic field model to determine the ionospheric footpoints and the IRI 90 ionosphere model values for the Pedersen conductance of $3.3 \mathrm{~S}$ and $8.4 \mathrm{~S}$ in the Northern and Southern Hemisphere, respectively, are found. The clear difference in hemispheric conductances indicates a clear shift of the null-point toward the Southern Hemisphere. This asymmetry of the Pg electric field variation may be described by a value $\alpha \approx 0.8$. Note, that $\alpha=1$ would correspond to a fundamental mode oscillation. With these estimates for $l / L$ and $\alpha$ we have $n \approx 0.4$.

The bounce frequency may be computed using the relation (Schulz and Lanzerotti, 1974)

$\Omega_{B}=\frac{\pi}{\sqrt{2 m_{p}} L R_{E}} \sqrt{W} \cdot \frac{1}{T(y)}$

where $m_{p}$ is the particle mass, $\mathrm{R}_{E}=6371 \mathrm{~km}$ the Earth radius, $L$ the McIlwain parameter, $W$ the particle energy, and $T(y)$ a factor taking into account the pitch angle $\alpha$ with $y=\cos \alpha$. For the pitch angles discussed here $T(y) \approx 1.3$. Thus with $W=67 \mathrm{keV}, \quad$ a value $\Omega_{B}=10^{-1} \mathrm{rads}^{-1}$ results. With $\omega=2 \pi / 100 \mathrm{~s}$, where $T=100 \mathrm{~s}$ is the observed giant pulsation period, and $\Omega_{D}=-(1.1 \pm 0.2) \times 10^{-3} \mathrm{rads}^{-1}$, the empirically determined channel 5 proton drift frequency, we receive azimuthal wave numbers

$$
\begin{aligned}
& m_{+}=-21 \pm 4 \\
& m_{-}=-93 \pm 20
\end{aligned}
$$


for $n= \pm 0.4$, respectively. The error estimate is based on the error in the determination of $\Omega_{D}$. Uncertainties in the determination of $n$ have not been considered. They will give rise to larger error limits for the wave numbers. Within the error limit the $n=0.4$-value of the azimuthal wave number, $m_{+}$, agrees with that one determined using ground-based magnetic field observations. This agreement strongly favours a drift-bounce resonance instability with $n \approx+0.4$ as a source mechanism for the observed giant pulsation. Any waves generated in $n \approx-0.4$ resonance would barely be observable at the ground due to the large $m$-value causing a large spatial attenuation of the wave signal below the ionosphere (e.g. Hughes and Southwood, 1976).

However, resonance condition (20) is only a necessary condition for instability. A more detailed investigation requires consideration of the physics of lowfrequency ring current instabilities. General approaches to this problem have been provided by Southwood (1976) and Chen and Hasegawa (1991). While Southwood (1976) has studied the problem using a driftkinetic approach, Chen and Hasegawa (1991) make use of the more detailed gyro-kinetic approximation. In the following we shall argue that the observed giant pulsation is generated by the unstable high-energy bump-on-the-tail part of the proton distribution. For these resonant protons and an azimuthal wave number of the order $|m|=30$ the ratio $k_{\perp} \rho \approx 0.1$ (where $k_{\perp}$ is the transverse wave number and $\rho$ the resonant proton gyroradius). Thus a drift-kinetic approximation as used by Southwood (1976) seems appropriate.

Southwood's (1976) general approach to low-frequency instabilities in the ring current provides an elegant framework for studying wave generation in the ring current. His central Eq. (22) may be written as (see also Chen and Hasegawa, 1991)

$\omega^{2} \delta K=2(\delta U+\delta M)+\mathrm{i} \omega \delta R$

where $\omega$ is the wave frequency, $\omega^{2} \delta K$ the change in flux tube integrated plasma kinetic energy, $\delta U$ the change in plasma bulk energy, and $\delta M$ the perturbation of magnetic energy. The term $i \omega \delta R$ describes energy changes due to resonant wave-particle interactions with $\delta R$ given in a dipole field topology by

$$
\begin{aligned}
\delta R & =\sum_{n} \int \mathrm{d} \tau \int 2 \pi^{2} \frac{|\vec{B}| \mathrm{d} \mu \mathrm{d} W}{v_{\|}}\left|\alpha_{n}\right|^{2} \\
& \times \delta\left(\omega_{r}-m \Omega_{D}-n \Omega_{B}\right) \frac{\mathrm{d} f}{\mathrm{~d} W} .
\end{aligned}
$$

Here the integration is over the flux tube volume defining the interaction region, $\vec{B}$ is the background magnetic field, $f$ the particle distribution function, $W$ the particle energy and $\mu$ its magnetic moment. Summation is over the different wave modes. The coefficient $\alpha_{n}$ describes the Fourier expansion of the particle bounce motion along field lines (Southwood, 1976). The delta-distribution describes the resonance character of the interaction.
Equations such as Eq. (23) are very suitable to analyse a plasma's stability. For example, without resonant particles $\delta R=0$, we have

$\omega^{2} \delta K=2(\delta U+\delta M)$

and the plasma is unstable, if $\delta U+\delta M<0$, as in this case $\omega$ becomes an imaginary number. The mirror and firehose modes are instabilities of this kind, i.e. they are purely growing modes (Kruskal and Oberman, 1958; Chen and Hasegawa, 1991). In more general cases with $\omega=\omega_{r}+\mathrm{i} \gamma$ from the imaginary part for the growth rate we assume:

$\gamma=\frac{\delta R}{2 \delta K}$.

Thus an instability may be driven by resonant waveparticle interaction, provided $\delta R>0$ and $\delta K>0$.

The original treatment by Southwood (1976) discusses the effect of an adiabatically injected background plasma population and concludes that mainly particles in $n=-1$ resonance destabilise the plasma, while the $n=+1$ particles have a stabilising influence. Thus, Southwood (1976) as well as Green (1979), Hughes et al. (1978), Glassmeier (1980), Poulter et al. (1983), or Chisham and Orr (1991) assumed that only even-mode harmonics with an asymmetric electric field variation can be excited. As some observations suggest that giant pulsations are odd-mode field line eigenoscillations (with symmetric electric field variation) the bounceresonance instability has been ruled out as a viable generation mechanism for these peculiar wave modes (Green 1976; Takahashi et al., 1992). However, in view of the clear correlation found between the October 4, 1978 giant pulsation event and substorm associated proton injection as well as the observation of a bumpon-the-tail distribution of the ring-current plasma a somewhat different interpretation emerges, if one carefully considers the assumptions on which the even-mode hypothesis is based.

In particular, Southwood (1976) assumed an adiabatically injected background plasma distribution as he is mainly interested in studying the stability of the background plasma against bounce resonance interactions. In the particular case we are analysing the background plasma distribution has been changed by substorm injected protons. The total distribution function may thus be approximated as

$f(\mu, W)=f_{B}(\mu, W)+f_{I}(\mu, W)$

where indices ' $B$ ' and ' $I$ ' denote the background distribution and the substorm injected particles, respectively. The gradient $\mathrm{d} f / \mathrm{d} W$ may be estimated as

$\left.\frac{\mathrm{d} f}{\mathrm{~d} W} \approx \frac{\partial f_{B}}{\partial W}\right|_{\mu, L}+\left.\frac{\partial f_{I}}{\partial W}\right|_{\mu, L}+\left.\frac{\partial L}{\partial W} \frac{\partial f_{B}}{\partial L}\right|_{\mu, W}$

Here, the variation of the substorm injected particle distribution $f_{I}(\mu, W)$ with radial distance has been neglected as we regard its effect in the following instability considerations as minor. 
Due to a lack of detailed information about the substorm injected distribution we assume that it varies with energy $W$ in a manner proportional to the variation of the background plasma. This ansatz

$\left.\frac{\partial f_{I}}{\partial W}\right|_{\mu, L}=\left.\eta \cdot \frac{\partial f_{B}}{\partial W}\right|_{\mu, L}$

for the phase space gradient of the bump-on-the-tail part of the proton distribution is very convenient as it allows formulation of the gradient in terms of the drift frequency $\Omega_{D}$ and via the resonance condition in terms of the bounce frequency $\Omega_{B}$, the mode number $n$, and the azimuthal wave number $m$. The proportionality between between the background and bump-on-the-tail parts is described by the factor $\eta$.

The phase space gradient for the resonant particles of the background plasma can be approximated as (Southwood et al., 1969)

$\frac{\partial f_{B}}{\partial W}=\left.\frac{n \Omega_{B}}{\omega_{r}} \frac{\partial f_{B}}{\partial W}\right|_{\mu, L}$,

and the total phase space gradient is approximately given by the expression,

$\left.\frac{\mathrm{d} f}{\mathrm{~d} W} \approx\left(\eta+\frac{n \Omega_{B}}{\omega_{r}}\right) \frac{\partial f_{B}}{\partial W}\right|_{\mu, L}$.

The growth rate Eq. (26) then reads

$$
\begin{gathered}
\gamma \delta K \approx \sum_{n} \int \mathrm{d} \tau \int \pi^{2} \frac{|\vec{B}| \mathrm{d} \mu \mathrm{d} W}{v_{\|}}\left|\alpha_{n}\right|^{2} . \\
\left.\delta\left(\omega_{r}-m \Omega_{D}-n \Omega_{B}\right)\left(\eta+\frac{n \Omega_{B}}{\omega_{r}}\right) \frac{\partial f_{B}}{\partial W}\right|_{\mu, L} .
\end{gathered}
$$

Under normal adiabatic conditions $\partial f_{B} / \partial W<0$, and the ring current plasma becomes unstable against resonant wave-particle interaction if

$\eta+\frac{n \Omega_{B}}{\omega_{r}}<0$

If there are no substorm injected particles present, i.e. $\eta=0$,

$\frac{n \Omega_{B}}{\omega_{r}}<0$

results, which gives one $n<0$ for instability. Particles in $n>0$ resonance are obviously stabilising the plasma. However, if $\eta \neq 0$, as expected for a bump-on-the-tail distribution, we have

$\eta<-n \frac{\Omega_{B}}{\omega_{r}}$

for instability. Particles in $n>0$ resonance now contribute to instability provided $|\eta|>\left|n \frac{\Omega_{B}}{\omega_{r}}\right|$, that is if the bump-on-the-tail distribution phase space gradient is sufficiently large. The interpretation of this finding is straightforward. Instability is due to two different gradients, the one in $L$-space and the one in $W$-space. With respect to the $L$-space gradient background plasma particles in $n>0$ resonance stabilise the plasma, while bump-on-the-tail $n>0$ resonant particles destabilise due to their positive $W$-space gradient. If the later particles outweigh the stabilising effect of the background particles, that is if the bump-on-the-tail is strong enough, the plasma becomes unstable.

In the present case $\omega_{r}=6.28 \times 10^{-2}$ rad $\mathrm{s}^{-1}$, $\Omega_{B} \approx 10^{-1} \mathrm{rad} \mathrm{s} \mathrm{s}^{-1}$, and $n \approx 0.45$. Thus, a value $\eta<-0.72$ would be sufficient for instability of the odd-harmonic such as the $n=0.45$ mode. Such a gradient is compatible with the GEOS-2 particle distribution observations. This discussion of the sufficient instability conditions therefore provides further support of a bump-on-the-tail driven bounce-resonance instability driving the observed $\mathrm{Pg}$.

A question remaining is the generation of the the unstable phase space density, that is the bump-on-thetail distribution causing $\partial f / \partial W>0$. Hughes et al. (1978) argue that unstable bump-on-the-tail distributions are naturally generated due to the combined action of gradient and curvature drift as well as $\vec{E} \times \vec{B}$ drift due to a solar wind induced dawn-dusk electric field. The $\vec{E} \times \vec{B}$ drift is essentially energy independant and larger than the gradient drift for energies below about $10 \mathrm{keV}$. Above $10 \mathrm{keV}$ ions gradient drift from the afternoon side westward via the frontside to the morning side (see Fig. 8). The $\vec{E} \times \vec{B}$ drift opposes the gradient drift of protons, giving rise to a velocity filter effect allowing only protons above $10 \mathrm{keV}$ to drift westward to the morning.

In the present case associated proton energies are much larger, and $\vec{E} \times \vec{B}$ drift effects are essentially unimportant. Velocity dispersion will lead to unstable distribution far away from the injection point as schematically shown in Fig. 8. Faster protons will reach a certain point earlier than lower energy particles. Highenergy particles are then added to the local background plasma at a higher rate than low-energy particles. This causes the build-up of the bump-on-the-tail distribution. This distribution, however, is not stable as lower energy particles follow up and the higher energy ones are moving further. Only if the dispersion is large enough and the injection of particles lasts for a long enough time

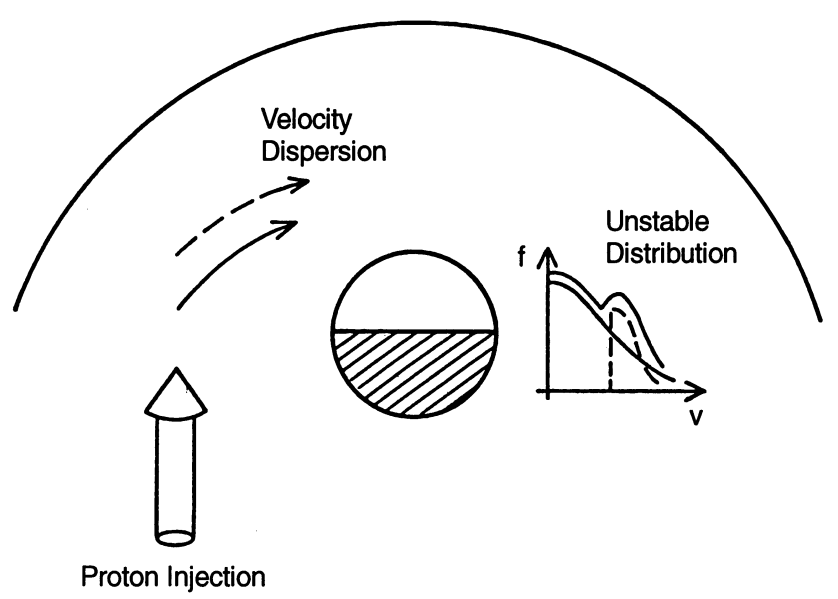

Fig. 8. Schematic representation of proton injection, velocity dispersion, and resulting unstable particle phase space density distribution in the dayside magnetosphere 
can an unstable distribution develop giving rise to the observed wave activity.

A detailed examination of how the unstable distribution evolves is beyond the scope of the present work and will be the subject of a future study on proton drifts in a realistic magnetic field geometry. However, several peculiarities of giant pulsations may be explained by this source hypothesis. For example, the instability can only set in at a great distance from the injection point, where the lifetime of the unstable distribution is larger than the inverse of the growth rate of the instability. The distribution is not only relaxing back to equilibrium by waveparticle interactions, but also due to the lower energy particles arriving and filling the low energy part of the phase space. This condition for the instability to occur fits very well with the observations that giant pulsations are usually observed in the early morning hours, that is at a great distance away from the proton injection point.

\section{Conclusions}

A ground-satellite coordinated case study of a giant pulsation event has been presented, which demonstrates that giant pulsations are associated with a drifting proton cloud injected into the nightside magnetospheric ring current due to substorm activity. The following conclusions are reached based on results from this study and previous work:

1. Substorm activity in the nightside magnetosphere causes injection of high-energy protons from the magnetotail into the eveningside magnetosphere.

2. Under the influence of gradient and curvature drifts these particles drift westward towards the dayside in the ring current regime. Velocity dispersion occurs, much as observed.

3 . This velocity dispersion can lead to bump-on-the-tail phase space distributions with the injected protons overlaying the ambient plasma. Further theoretical work and numerical studies are required to follow the evolution of the injected particle population in detail. It may be speculated that dispersion must be large enough to result in distributions unstable against wave-particle interaction. The predominantly morningside occurrence of Pg's could be explainable in this way.

4. The bump-on-the-tail distribution causes a positive phase space density gradient, that is $\partial f / \partial W>0$. Under assumptions discussed in more detail earlier this positive gradient causes the plasma to become unstable against the excitation of odd-mode type fundamental field line eigenoscillations via a drift-bounce resonance. The conclusion of an odd-mode being excited differs from previous studies (e.g. Southwood, 1976; Chen and Hasegawa, 1991) where the unstable plasma has been assumed to be an adiabatically generated type with $\partial f / \partial W<0$. The addition of particles injected due to substorm activity changes the phase space gradient and allows the odd-mode to become unstable. For the first time this odd-mode structure has been confirmed observationally for the October 4, 1978 event, discussed in this study.
5. A resonant wave-particle interaction also allows us to understand the typical quasi-monochromatic wave form of Pgs. Furthermore, resonance requires a significant Doppler shift, which causes a large azimuthal wave number, $m$, of any unstable wave. Observed and theoretically deduced $m$-values for the event under discussion agree fairly well.

6. A large azimuthal wave number, about $m=-26$ in our case, requires the excited wave to propagate as a guided poloidal mode. These modes have a predominantly radial magnetic field perturbation as first discussed by Dungey (1954). A radial magnetic field variation is associated with an azimuthal electric field oscillation. The observed electric field perturbation at GEOS-2 is mainly in the azimuthal direction, in accord with the hypothesis. Furthermore, at the ground a magnetic field oscillation is expected which is rotated by about $90^{\circ}$ against that in the magnetosphere (e.g. Hughes, 1974; Glassmeier, 1984). Thus the Pg oscillation should be largest in the D component at the ground. This is as observed.

Finally, we would like to mention that giant pulsations are evidence of a very fundamental process for the generation of unstable particle phase space distributions in the Earth's magnetosphere.

Acknowledgements. We are grateful to Gordon Rostoker for providing us with Fort Providence magnetic field observatories. The work by K.H.G. and S.B. was supported by the Deutsche Forschungsgemeinschaft and the German Space Agency DARA (now DLR), the work by A.K. by the German Bundesministerium für Wissenschaft und Technologie, and the work of U.M. through a Werner-Heisenberg-Stipendium of the Deutsche Forschungsgemeinschaft. Support from Frank Budnik, Stefan Markgraf and Nicole Cornilleau-Wehrlin is gratefully acknowledged.

The Editor in chief thanks T.K Yeoman and another referee for their help in evaluating this paper.

\section{References}

Allan, W., Phase variation of ULF pulsations along the geomagnetic field line, Planet. Space Sci., 30, 339-346, 1982.

Allan, W., and F. B. Knox, Interpretation of an ATS-6 Alfvén wave using solutions with finite ionospheric conductivity, Geophys. Res. Lett., 5, 849-852, 1978.

Allan, W., and F. B. Knox, A dipole field model for axisymmetric Alfvén waves with finite ionospheric conductivities, Planet. Space Sci., 27, 79-91, 1979.

Annexstad, J. O., and C. R. Wilson, Characteristics of $\mathrm{Pg}$ micropulsations at conjugate points, J. Geophys. Res., 73, 1805-1818, 1968.

Candidi, M., R. Orfei, F. Paluten, and G. Vannaroni, FFT analysis of space magnetometer noise, IEEE Trans, Geosci. Electron., GE-12, 23-28, 1974.

Chen, L., and A. Hasegawa, Kinetic theory of geomagnetic pulsations. 1. Internal excitations by energetic particles, J. Geophys. Res., 96, 1503-1511, 1991.

Chisham, G., Giant pulsations (Pgs): an explanation for their rarity and occurrence during geomagnetically quiet times, J. Geophys. Res., 101, 24755, 1996.

Chisham, G., and D. Orr, Statistical studies of giant pulsations (Pgs): harmonic mode, Planet. Space Sci., 39, 999-1020, 1991.

Chisham, G., and D. Orr, The association between giant pulsations (Pgs) and the auroral oval, Ann. Geophysicae., 12, 649, 1994.

Chisham, G., D. Orr, and T.K. Yeoman, Observations of a giant pulsation $(\mathrm{Pg})$ across an extended array of ground magnetometers and an auroral radar, Planet. Space Sci., 40, 953-964, 1992. 
Chisham, G. I. R. Mann, and D. Orr, A statistical study of giant pulsation ( $\mathrm{Pg}$ ) latitudinal polarization and amplitude variation, J. Geophys. Res., 102, 9619, 1997.

Cummings, W. D., R. J. O'Sullivan, and P. J. Coleman, Standing Alfvén waves in the magnetosphere, J.Geophys. Res., 74, 778791,1969

Dungey, J. W., Electrodynamics of the outer atmosphere, Penn. State University, Ionos. Res. Lab. Sci. Rep., 69, 1954.

Glassmeier, K. H., Magnetometer array observations of a giant pulsation event, J. Geophys., 48, 138-147, 1980.

Glassmeier, K. H., On the influence of ionospheres with nonuniform conductivity distribution on hydromagnetic waves, J. Geophys., 54, 125-138, 1984.

Glassmeier, K. H., M. Lester, W. A. C. Mier-Jedrzejowicz, C. A. Green, G. Rostoker, D. Orr, U. Wedeken, H. Junginger, and E. Amata, Pc5 pulsations and their possible source mechanisms: a case study, J. Geophys., 55, 108-119, 1984.

Glassmeier, K. H., M. v. Seggern, R. v. Stein, and A. Korth, Die Protonen Sprung Resonanz Instabilität als möglicher Anregungsmechanismus von Riesenpulsationen, Kleinheubacher Ber., 35, 217-221, 1992.

Green, C. A. Observations of Pg pulsations in the northern auroral zone and at lower latitude conjugate regions, Planet. Space Sci., 27, 63-77, 1979.

Hasegawa, A., Drift-wave instabilities of a compressional mode in a high-beta plasma, Phys. Rev. Lett., 27, 11-14, 1971.

Hughes, W. J., The effect of the atmosphere and ionosphere on long-period magnetospheric micropulsations, Planet. Space Sci., 22, 1157-1173, 1974.

Hughes, W. J., and D.J. Southwood, The screening of micropulsation signals by the atmosphere and ionosphere, J. Geophys. Res., 81, 3234-3242, 1976.

Hughes, W. J., D. J. Southwood, and B. Mauk, Alfvén waves generated by an inverted plasma energy distribution, Nature, 275, 43-44, 1978.

Korth, A., and B. Wilken, New magnetic electron spectrometer with directional sensitivity for a satellite application, Rev. Sci. Instrum., 49-61, 1435, 1978.

Korth, A., G. Kremser, and B. Wilken, Observations of substorm associated particle-flux variations at $6<\mathrm{L}<8$ with GEOS-1, Space Sci. Rev., 22, 501-509, 1978.
Kruskal, M. D., and C. R. Oberman, On the stability of plasma in static equilibrium, Phys. Fluids, 1, 275-280, 1958.

Küppers, F., J. Untiedt, W. Baumjohann, K. Lange, and A. G. Jones, A two-dimensional magnetometer array for ground-based observations of auroral zone electric currents during the International Magnetospheric Study (IMS), J. Geophys., 46, 429-450, 1979.

Poulter, E. M., W. Allan, E. Nielsen, and K.H. Glassmeier, STARE radar observations of a Pg pulsation, J. Geophys. Res., 88, 5668-5673, 1983.

Roederer, J. G., Dynamics of geomagnetically trapped radiation, Springer-Verlag, Berlin Heidelberg New York 1979.

Rostoker, G., H. L. Lam, and J. V. Olson, Pc 4 giant pulsations in the morning sector, J. Geophys. Res., 84, 5133-5166, 1979.

Schulz, M., The magnetosphere, in Ed. J. A. Jacobs, Geomagnetism, vol 4, p. 203, Academic Press, London, 1991.

Schulz, M., and L. J. Lanzerotti, Particle diffusion in the radiation belts, Springer-Verlag, Berlin, Heidelberg, New York, 1974.

Singer, H. J., C. T. Russell, M. G. Kivelson, T. A. Fritz, W. Lennartsson, Satellite observations of the spatial extent and structure of $\mathrm{Pc} 3,4,5$ pulsations near the magnetospheric equator, Geophys. Res. Lett., 11, 889-892, 1979.

Southwood, D. J., A general approach to low-frequency instability in the ring current plasma, J. Geophys. Res., 81, 3340, 1976.

Southwood, D. J., and M. G. Kivelson, Charged particle behavior in low-frequency geomagnetic pulsations. 2. Graphical approach, J. Geophys. Res., 87, 1707-1710, 1982.

Southwood, D. J., J. W. Dungey, and R. J. Etherington, Bounce resonant interaction between pulsations and trapped particles, Planet. Space Sci., 17, 349-3561, 1969.

Takahashi, K., N. Sato, J. Warnecke, H. Lühr, H. E. Spence, and Y. Tonegawa, On the standing wave mode of giant pulsations, J. Geophys. Res., 97, 10717-10732, 1992.

Wedeken, U., B. Inhester, A. Korth, K. H. Glassmeier, R. Gendrin, L. J. Lanzerotti, H. Gough, C. A. Green, E. Amata, A. Pedersen, G. Rostoker, Ground satellite coordinated study of the April 5, 1979 events: flux association of energetic particles and associated magnetic pulsations, J. Geophys., 55, 120-133, 1984. 